\title{
Management and prognostic prediction of appendiceal mucinous adenocarcinoma with peritoneal metastasis: a single center study in China
}

\author{
Ruiqing Ma', Bing Wang ${ }^{1}$, Xichao Zhai ${ }^{1}$, Yiyan $\mathrm{Lu}^{2}$ and Hongbin Xu ${ }^{1 *}$
}

\begin{abstract}
Background: To investigate the clinical and pathological characteristics of appendiceal mucinous adenocarcinoma with peritoneal metastasis and analyze the prognostic factors.

Methods: A retrospective analyses of clinicopathological features of 50 patients with appendiceal mucinous adenocarcinoma with peritoneal metastasis from January, 2013 to December, 2017 in Aerospace Central Hospital, Beijing, China. Survival data calculation and comparison were respectively performed with the Kaplan-Meier method and the log-rank test. The Cox proportional hazards regression method was used for multivariate survival analyses.

Results: Cytoreduction for appendiceal mucinous adenocarcinoma was conducted on 50 patients (24 males and 26 females), with a median age of 52.5 years at the time of surgery (range 31-71 years). The median overall survival (OS) time was 24 months, with 2-3- and 5-year survival rates of 53, 24 and 8\%, respectively. At the last follow-up in December 2018, 13 patients were still alive. Multivariate analysis revealed that patients who had low Ki-67 expression (less than 50\%) and CCR (completeness of cytoreduction) 0/1/2 score had significantly better OS rate than their respective counterparts.
\end{abstract}

Conclusions: Ki-67 expression statue and CCR score could be employed as the prognosis prediction in patients with appendiceal mucinous adenocarcinoma.

Keywords: Appendiceal mucinous adenocarcinoma, Pseudomyxoma peritonei, Completeness of cytoreduction score, Prognosis

\section{Introduction}

Malignant appendix neoplasms are extremely rare and the incidence of primary appendiceal cancers was less than $1.5 \%$ (approximately 0.12 per $1,000,000$ person years) according to the appendectomy specimens examination [1-3]. As the primary malignant neoplasms of the appendix, appendix adenocarcinomas includes mucinous, non-mucinous (colonic-type), and signet-ring

\footnotetext{
* Correspondence: 501918613@qq.com

'Department of Myxoma, Aerospace Center Hospital, Beijing 100049, China Full list of author information is available at the end of the article
}

cell adenocarcinomas [4]. Among them, mucinous adenocarcinoma is the most common type. Since appendix carcinomas may cause appendicitis or the appendix rupture, and pseudomyxoma peritonei (PMP) could be presented as a typical clinical syndrome when mucinous adenocarcinoma of the appendix spreads to the peritoneal cavity, which is characterized by a large amount recurrent and recalcitrant mucinous ascites caused by surface growth on the peritoneum without obvious underlying tissues invasion.

(c) The Author(s). 2020 Open Access This article is licensed under a Creative Commons Attribution 4.0 International License, which permits use, sharing, adaptation, distribution and reproduction in any medium or format, as long as you give appropriate credit to the original author(s) and the source, provide a link to the Creative Commons licence, and indicate if changes were made. The images or other third party material in this article are included in the article's Creative Commons licence, unless indicated otherwise in a credit line to the material. If material is not included in the article's Creative Commons licence and your intended use is not permitted by statutory regulation or exceeds the permitted use, you will need to obtain permission directly from the copyright holder. To view a copy of this licence, visit http://creativecommons.org/licenses/by/4.0/ The Creative Commons Public Domain Dedication waiver (http://creativecommons.org/publicdomain/zero/1.0/) applies to the data made available in this article, unless otherwise stated in a credit line to the data. 
According to the 8th edition of the American Joint Committee on Cancer (AJCC) Staging Manual, mucinous adenocarcinomas of the appendix are classified into low-grade and high-grade (well-differentiated and moderately/poorly differentiated, respectively) tumor based on histological grade in the AJCC TNM Staging System [5-7]. Due to the low incidence, lacking of available data about the prognostic factors for appendix mucinous adenocarcinomas and non-adenocarcinomas in current clinical practice.

Here, we presented our single center data about the clinical and pathological characteristics of appendiceal mucinous adenocarcinoma and analyze the prognostic factors by retrospective review of 50 cases of patients.

\section{Patients and methods}

\section{Ethical approval of the study}

The study was approved by the Ethic committee of Aerospace Central Hospital, Beijing, China (no.20161109-ST07). Written inform consent was obtained from all the included patients, and all the participants have given the consent for their images.

\section{Patients}

A retrospective analyses of a cohort of appendiceal mucinous adenocarcinoma patients with peritoneal metastasis from January, 2013 to December, 2017 in the Department of Myxoma of Aerospace Central Hospital, Beijing, China. All the patients were screened from high grade peritoneal pseudomyxoma and confirmed by 2 experienced pathologists. Mucinous adenocarcinoma was featured by invasive peritoneal lesions comprised with abundant glandular or signet ring cell morphology epithelium with sufficient architectural complexity and cytological atypia to a diagnosis confirmation of mucinous carcinoma. The diagnosis criteria were according to the 8th Edition of American Joint Committee on Cancer [7]. The exclusion criteria were as follows: 1. Lost follow-up; 2 combined with other severe organic disease; 3. with incomplete disease history. Finally, a total of 50 patients were included for analyses.

\section{Surgical procedure}

Sugarbaker technique of cytoreductive surgery (CRS)/ hyperthermic intraperitoneal chemotherapy (HIPEC) described previously were employed here [8]. Briefly, aggressive cytoreduction was employed to remove as much macroscopic disease as possible. HIPEC is administered at thecompletion of cytoreduction using closed technique. Two inflow and two outflow catheters were allocated in the abdomen. After temporary the abdominal skin closure, mitomycin at the dosage of $20 \mathrm{mg} / \mathrm{m}^{2}$ was performed to increase to an inflow temperature of 41 to $42{ }^{\circ} \mathrm{C}$ and follow by a 60 min' peritoneal cavity circulation (Fig. 1).

\section{Data collection}

The interested variables included age of the patient at the time of diagnosis, sex, Prior surgical score (PSS), with/without preoperative intravenous chemotherapy, preoperative intraperitoneal chemotherapy, hemoglobin levels, albumin levels at the time of admission, preoperative CEA/CA125/CA199 elevation, Intraoperative peritoneal cancer index (PCI), completeness of cytoreduction (CCR) score, intraoperative HIPEC; pathological data include: with/without lymph node metastasis, presence or absence of signet ring cells. Among these characteristics, PSS was scored 0-3, and PSS-0: biopsy only, PSS-1: minimal prior dissection with only one dissected abdominal region; PSS-2: 2-5 regions dissected and PSS-3: extensive prior cytoreduction (more than 5 regions). PCI stands for a clinical integration of both peritoneal implant size and distribution of peritoneal surface malignancy [9]. For PCI evaluation, 13 anatomical regions were divide based on the region of the abdomen and pelvis. The largest size malignant nodule at each region was scored and the lesion size score from the
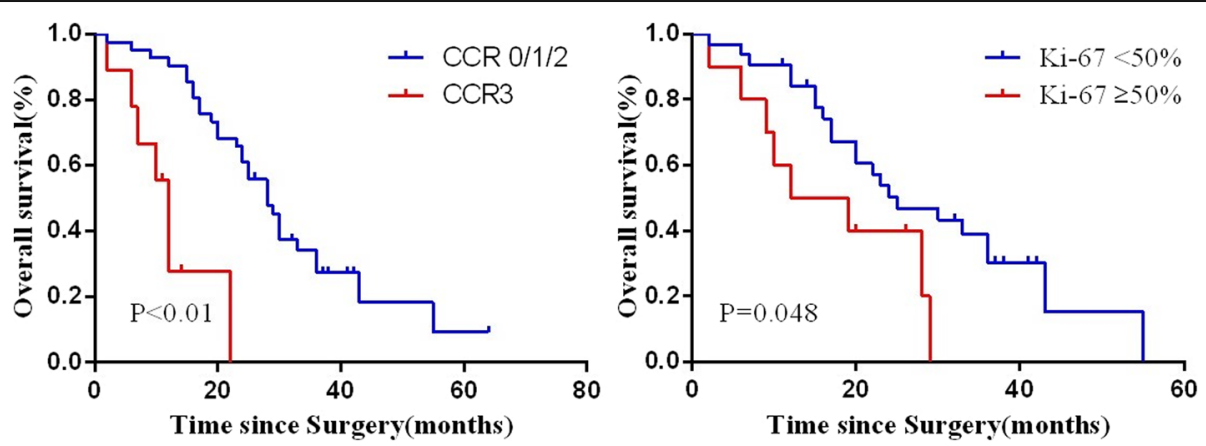

Fig. 1 Surgical procedure and pathological analysis of appendiceal mucinous adenocarcinoma. a. Mucinous ascites in peritoneal cavity (black arrow); b. Great omentum cake in peritoneal cavity; $\mathbf{c}$. The status of abdominal cavity after cytoreduction therapy; $\mathbf{d}$. CT scan at the time of precytoreduction therapy (black arrow indicated the lesion); e. CT scan image after cytoreduction therapy (black arrow indicated no lesion postsurgery); f. H\&E staining of tissue sections of appendiceal mucinous adenocarcinoma 
all 13 regions was summated as the PCI for the individual patient [8]. CCR score was recorded at the time of the completion of the surgical procedure. CCR-0: no peritoneal seeding was visible after the cytoreduction, CCR-1: residual tumour nodules less than $2.5 \mathrm{~mm}, \mathrm{CCR}-2$ : residual tumour nodules $2.5 \mathrm{~mm}$ to $2.5 \mathrm{~cm}$ and a CCR-3: larger than $2.5 \mathrm{~cm}$ persistent tumor nodules [10].

\section{Statistical analyses}

Statistical analyses were employed using SPSS 20.0(SPSS Inc. Chicago, IL). Continuous data were expressed as medians and range. Categorical variables were presented as number and percentages. The associations between the clinicopathological variables and survival data was identified by univariate and multivariate Cox proportional hazard regression models. All variables with $p$ value $<0.3$ in the univariate analysis were further analyzed with in the multivariate Cox model. Survival data calculation and comparison were respectively performed with the Kaplan-Meier method and the log-rank test. Statistically significance was considered when the presence of two tailed $P$ values $<0.05$.

\section{Results}

Demographic and pathological characteristics of patients

Cytoreductive surgery for appendiceal mucinous adenocarcinoma was carried out on 50 patients (26 males and 24 females), with a median age of 52.5 years at the time of surgery (range 31-71 years). The median survival rate was 24 months, whereas 2-,3- and 5-year survival rates of 53,24 and $8 \%$, respectively. Prior systemic chemotherapy was performed in $13(26 \%)$ patients, whereas $15(30 \%)$ patients were treated with HIPEC. Forty-eight (96\%) patients were with bloating or ascites or abdominal mass manifestation. Comorbidities including ileus, anemia and hypoalbuminemia were respectively found in $18(36 \%)$, 23(46\%) and 9(18\%) patients. Increased levels of Serum tumor maker CEA, CA125 and CA199 were respectively in $33(66 \%), 34(68 \%)$ and $27(54 \%)$ patients. The number of patients with PCI score $>26,13-26$, and $<13$ were respectively $34(68 \%), 14(28 \%)$ and $2(4 \%)$. Demographic and pathological characteristics of patients were listed in Table 1.

\section{Multivariable cox regression analysis for prognosis prediction}

According to the multivariate analysis, significantly better overall survival rate was found in the patients with a low Ki-67 expression (less than 50\%) and CCR0/1/2 score than their corresponding counterparts (Table 2).

\section{Survival}

At the last follow-up in December 2018, 13 patients were still alive. The median overall survival (OS) time
Table 1 Patient demographic data $(n=50)$

\begin{tabular}{|c|c|}
\hline \multicolumn{2}{|l|}{ Characteristics } \\
\hline \multicolumn{2}{|l|}{ Gender } \\
\hline Female & $24(48 \%)$ \\
\hline Male & $26(52 \%)$ \\
\hline Disease duration (median; range; months) & $18(1-357)$ \\
\hline \multicolumn{2}{|l|}{ PSC } \\
\hline No & $37(74 \%)$ \\
\hline Yes & $13(26 \%)$ \\
\hline \multicolumn{2}{|l|}{ PPC } \\
\hline No & $35(70 \%)$ \\
\hline Yes & $15(30 \%)$ \\
\hline \multicolumn{2}{|l|}{ Manifestation at diagnosis } \\
\hline Bloating or ascites or abdominal mass & $48(96 \%)$ \\
\hline Others & $2(4 \%)$ \\
\hline \multicolumn{2}{|l|}{ Comorbidities } \\
\hline ileus & $18(36 \%)$ \\
\hline anemia & $23(46 \%)$ \\
\hline hypoalbuminemia & $9(18 \%)$ \\
\hline \multicolumn{2}{|l|}{ Increased levels of Serum tumor maker } \\
\hline CEA & $33(66 \%)$ \\
\hline CA125 & $34(68 \%)$ \\
\hline CA199 & $27(54 \%)$ \\
\hline \multicolumn{2}{|l|}{$\mathrm{PCl}$} \\
\hline$<13$ & $2(4 \%)$ \\
\hline $13-26$ & $14(28 \%)$ \\
\hline$>26$ & $34(68 \%)$ \\
\hline \multicolumn{2}{|l|}{ CCR } \\
\hline 0,1 & $20(40 \%)$ \\
\hline 2 & $21(42 \%)$ \\
\hline 3 & $9(18 \%)$ \\
\hline \multicolumn{2}{|l|}{ HIPEC } \\
\hline No & $9(18 \%)$ \\
\hline Yes & $41(82 \%)$ \\
\hline \multicolumn{2}{|l|}{ Signet ring cell } \\
\hline No & $42(84 \%)$ \\
\hline Yes & $8(16 \%)$ \\
\hline \multicolumn{2}{|l|}{ Lymph node metastasis } \\
\hline No & $43(86 \%)$ \\
\hline Yes & $7(14 \%)$ \\
\hline \multicolumn{2}{|l|}{ Ki-76 } \\
\hline$<50$ & $32(64 \%)$ \\
\hline$\geq 50$ & 18(36\%) \\
\hline Follow-up time (median; range; months) & $24(12-36)$ \\
\hline
\end{tabular}

PSC Previous systemic chemotherapy, PPC previous peritoneal chemotherapy, $P C l$ peritoneal cancer index, CCR completeness of cytoreduction, HIPEC Hyperthermic intraperitoneal chemotherapy 
Table 2 Univariate and multivariable Cox Proportional Hazards Regression Model for prognosis prediction in patients with appendiceal mucinous adenocarcinoma

\begin{tabular}{|c|c|c|c|c|}
\hline Variable & Univariate hazard ratio(range) & $P$ value & Multivariate hazard ratio(range) & $P$ value \\
\hline Age (> 60 vs. $\leq 60)$ & $1.059(0.545-2.056)$ & 0.867 & & \\
\hline Gender & $1.334(0.683-2.603)$ & 0.399 & & \\
\hline PSS & $0.898(0.663-1.216)$ & 0.487 & & \\
\hline CA125 & $1.873(0.877-4.000)$ & 0.105 & & \\
\hline PSC & $0.573(0.249-1.319)$ & 0.191 & & \\
\hline Ileus & $0.703(0.342-1.445)$ & 0.338 & & \\
\hline $\mathrm{PCl}$ & $1.836(0.942-3.576)$ & 0.074 & & \\
\hline CCR $(0,1,2$ vs.3) & $6.962(2.578-18.800)$ & $<0.001$ & 9.785 (3.381-28.318) & $<0.001$ \\
\hline HIPEC & $0.758(0.314-1.832)$ & 0.538 & & \\
\hline Ki-67 expression ( $<50 \%$ vs. $\geq 50 \%$ ) & $1.715(0.856-3.438)$ & 0.128 & $2.356(1.125-4.931)$ & 0.023 \\
\hline Signet ring Cell & $2.073(0.895-4.802)$ & 0.089 & & \\
\hline Lymph node metastasis & $1.874(0.638-5.507)$ & 0.253 & & \\
\hline
\end{tabular}

PSS prior surgical score, PSC Previous systemic chemotherapy, PCI peritoneal cancer index, CCR completeness of cytoreduction, HIPEC Hyperthermic intraperitoneal chemotherapy

was 24 months, while the 2-, 3- and 5-year survival rates of 53,24 and $8 \%$, respectively. The 1-year, 2-year survival rates for CCR0/1/2 and CCR3 were $90 \%$ vs $28,66 \%$ vs $0 \%$, respectively. The 1-year, 2-year survival rates for Ki- $67<50 \%$ and Ki- $67 \geq 50 \%$ were $67 \%$ vs $50,39 \%$ vs $0 \%$, respectively (Fig. 2).

\section{Discussion}

In present study, we performed a retrospective analysis of clinicopathological features of 50 patients with appendiceal mucinous adenocarcinoma to investigate the possible prognosis related factors. Our results indicated that cytoreduction for appendiceal mucinous adenocarcinoma was conducted on 50 patients (24 males and 26 females), with a median age of 52.5 years at the time of surgery (range $31-71$ years).

Immediate progression was considered in the majority of patients who underwent debulking surgery (CCR2/3). Only 20 patients had CCR0/1 cytoreduction. So, progression-free survival was not discussed in this study. The median overall survival (OS) time was 24 months, while the 2-, 3- and 5year survival rates of 53,24 and $8 \%$, respectively. At the last

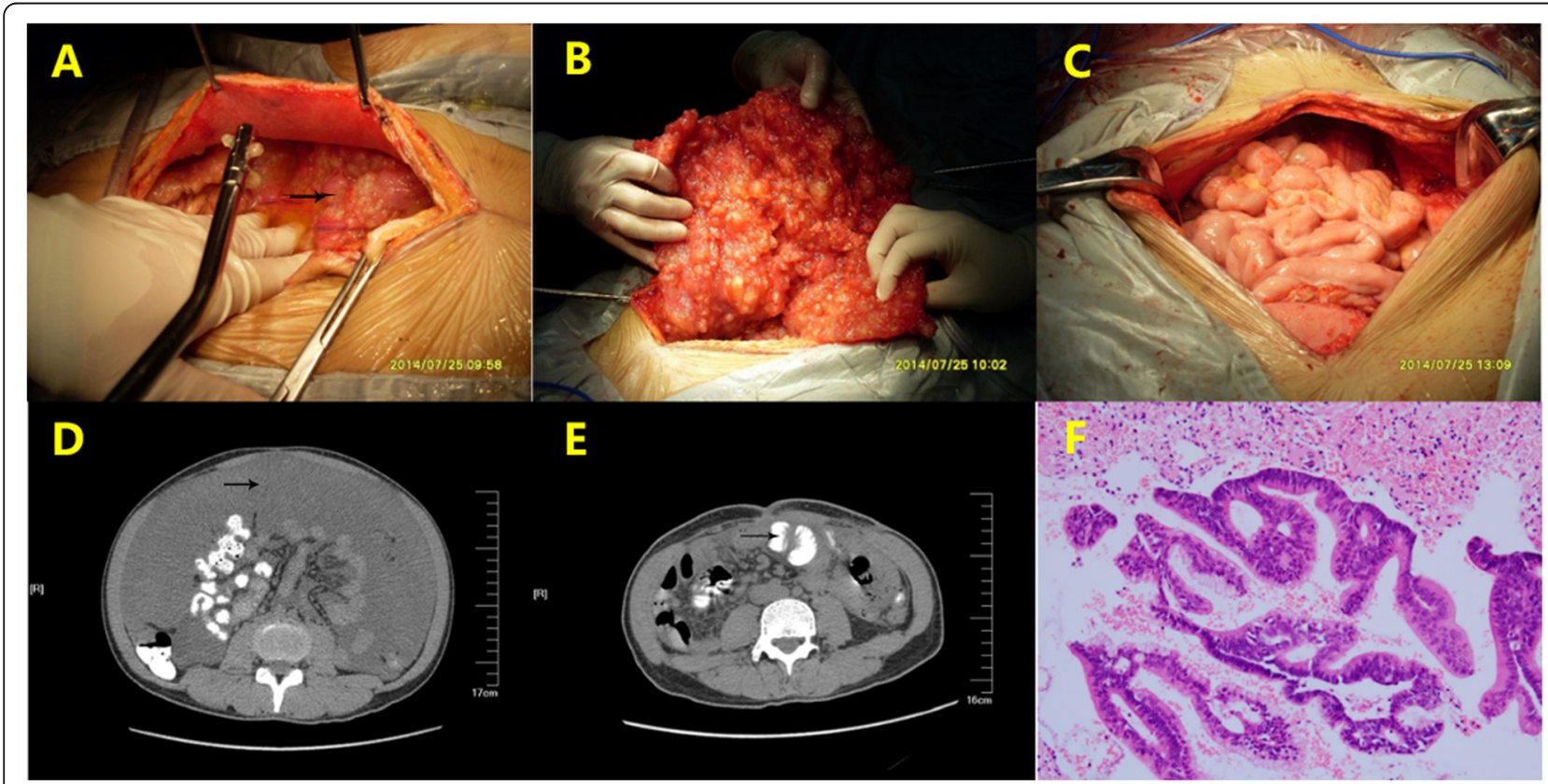

Fig. 2 Kaplan-Meier survival curve according to the completeness of cytoreduction (CCR) classification and Ki-67 classification 
follow-up in December 2018, 13 patients were still alive. Multivariate analysis revealed that patients who had less than 50\% Ki-67 expression and CCR0/1/2 score had significantly better OS rate than their respective counterparts.

Mucinous adenocarcinomas are considered as the unique appendix tumours based on their particular biological behavior. Among different types of appendiceal neoplasms, greater potential of the presence of serosa invasion and peritoneum or abdominal cavity spreading and followed by PMP formation was found in the mucinous adenocarcinomas. As a rare clinical disease, the characteristics of PMP include excessive accumulation of gel-like mucinous peritoneal fluid in the peritoneal or pelvic cavity, thereby resulting in clinical manifestation such as abdominal pain, abdominal mass, progressive increasing of the abdominal circumference and severe weight loss $[11,12]$. Variable results were found on the survival rate of patients with mucinous appendiceal adenocarcinoma according to previous reports [13-15]. Overman et al. found that stage IV diseases were more likely to be presented as mucinous adenocarcinomas than those with non-mucinous adenocarcinomas [16]. Therefore, the subdivision of the patients into Stage I-III and IV was conducted by Xie et al., and their results showed that significantly decreased 5-year overall survival was found in patients with mucinous adenocarcinoma, which indicated the different biological behaviors between mucinous and non-mucinous adenocarcinomas.

Multidisciplinary therapies employing surgical resection followed by adjuvant chemoradiation treatment, have been increasingly performed as the treatment modality in patients with resectable digestive tract cancers $[17,18]$. However, controversy was remained existed about whether the multidisciplinary therapies could result in a survival improve effects in patients with adenocarcinoma of the appendix. Since the development of PMP was the frequently presented complications in patients with mucinous adenocarcinomas of the appendix could, cytoreductive surgery and HIPEC have been suggested as first-line therapies [19]. Recently, Asare et al demonstrated that systematic chemotherapy could lead to a significantly improved OS without considering the history feature of the adenocarcinoma (HR:0.79; 95\% CI: 0.69-0.90; $P=0.0005$ and HR:0.84; 95\% CI: 0.75-0.95; $P=0.004$ for mucinous and nonmucinous, respectively). Moreover, systemic chemotherapy seems to have no effects on the patients with mucinous and welldifferentiated adenocarcinomas [20, 21]. In our study, multivariate analysis revealed that patients with CCR0/1/ 2 score had significantly improved 5-year overall survival rate than those with CCR3, whereas The OS times for the cohort based on CCR score of tumors were 28 months and 12 months, respectively, for CCR0/1/2, and CCR3. These results also supported the effectiveness of
HIPEC in the treatment of mucinous appendicular adenocarcinoma.

There are also some limitations in our study. Firstly, this is a single center small size study and insufficient sample size could affect the final conclusion. Secondly, the diagnosis value of $\mathrm{Ki}-67$ expression was also implicated by the results obtained here, and although we calculated accurate cut-off value of Ki-67 level by ROC curve, lacking of comparison results with golden standard could result in failure to elucidate the diagnostic efficacy of Ki-67. Third, the detailed mechanisms that resulted in the efficacy of CRS should be elucidated for identification of the exact role of CRS in mucinous appendicular adenocarcinoma. Taken together, further investigation is still important to identify the biomarker role of CCR scores by a multicenter clinical large sample size study with prognosis results.

\section{Conclusions}

In conclusions, we demonstrated that $\mathrm{Ki}-67$ expression statue and CCR score could be employed as the prognosis prediction in patients with appendiceal mucinous adenocarcinoma. Any effort to achieve CCR0/1/2 by operation is valuable to improve the prognosis. Patients with high volume disease such as appendiceal mucinous adenocarcinoma should be examined in a specialized center for evaluation based on the consideration of the potential survival benefit that may be achieved after cytoreduction.

\section{Abbreviations}

OS: overall survival; CCR: completeness of cytoreduction;

PMP: pseudomyxoma peritonei; AJCC: American Joint Committee on Cancer; CRS: cytoreductive surgery; HIPEC: hyperthermic intraperitoneal

chemotherapy; PSS: prior surgical score; PCl: peritoneal cancer index

\section{Acknowledgements}

We thank Shilong Wang for funding the data collection and database management. We also thank Aerospace Central Hospital for patient referral.

\begin{abstract}
Authors' contributions
The author contributions were as follows: RM, BW, and HX conceived and designed the experiments; RM, BW, $X Z, Y L$ and $H X$ performed the experiments; RM, BW, XZ, YL and $H X$ analyzed the data; RM, BW, XZ, YL and $\mathrm{HX}$ contributed reagents/materials/analysis tools; RM, BW, XZ, YL and HX contributed to the writing of the manuscript. All authors approved the final version of the manuscript to be submitted. All authors agreed to be accountable for all aspects of the work and ensuring that questions related to the accuracy or integrity of any part of the Article are appropriately investigated and resolved.
\end{abstract}

\section{Authors' information}

No applicable.

\section{Funding}

No applicable.

Availability of data and materials

The datasets used and/or analyzed during the current study are available from the corresponding author on reasonable request. 


\section{Ethics approval and consent to participate}

This is a retrospective study, and all the patients signed the consent for treating before the treatment. Additionally, the participants have given the consent for their images. The study was approved by the Ethic committee of Aerospace Central Hospital, Beijing, China (no.20161109-ST-07).

\section{Consent for publication}

Written informed consent was given by the participants, and all the participants have given the consent for their images.

\section{Competing interests}

The authors declare no conflict of interest.

\section{Author details}

'Department of Myxoma, Aerospace Center Hospital, Beijing 100049, China. 2Department of Pathology, Aerospace Center Hospital, Beijing, China.

Received: 23 September 2019 Accepted: 25 March 2020

Published online: 06 April 2020

\section{References}

1. Collins DC. 71,000 human appendix specimens. A final report, summarizing forty years' study. Am J Proctol. 1963;14:265-81.

2. Connor SJ, Hanna GB, Frizelle FA. Appendiceal tumors: retrospective clinicopathologic analysis of appendiceal tumors from 7,970 appendectomies. Dis Colon Rectum. 1998:41(1):75-80.

3. McCusker ME, Cote TR, Clegg LX, Sobin LH. Primary malignant neoplasms of the appendix: a population-based study from the surveillance, epidemiology and end-results program, 1973-1998. Cancer. 2002;94(12): 3307-12.

4. Ruoff C, Hanna L, Zhi W, Shahzad G, Gotlieb V, Saif MW. Cancers of the appendix: review of the literatures. ISRN Oncol. 2011;2011:728579.

5. Egner JR. AJCC cancer staging manual. JAMA. 2010;304(15):1726-7.

6. Padmanaban V, Morano WF, Gleeson E, Aggarwal A, Mapow BL, Stein DE, Bowne WB. Incidentally discovered low-grade appendiceal mucinous neoplasm: a precursor to pseudomyxoma peritonei. Clin Case Rep. 2016; 4(12):1112-6.

7. Keung EZ, Gershenwald JE. The eighth edition American Joint Committee on Cancer (AJCC) melanoma staging system: implications for melanoma treatment and care. Expert Rev Anticancer Ther. 2018;18(8):775-84.

8. Sugarbaker PH. Peritonectomy procedures. Ann Surg. 1995;221(1):29-42.

9. Sugarbaker PH. Management of peritoneal surface malignancy using intraperitoneal chemotherapy and cytoreductive surgery: manual for physicians and nurses; 1998.

10. Sugarbaker PH. Successful management of microscopic residual disease in large bowel cancer. Cancer Chemother Pharmacol. 1999;43(Suppl): S15-25.

11. Ramaswamy V. Pathology of mucinous Appendiceal tumors and Pseudomyxoma Peritonei. Indian J Surg Oncol. 2016;7(2):258-67.

12. Wang $H$, Wang $X$, Ju $Y$, Wang J, Zhang $X$, Cheng $Y$, Sun J, Hu Y. Clinicopathological features and prognosis of pseudomyxoma peritonei. Exp Ther Med. 2014;7(1):185-90.

13. Nitecki SS, Wolff BG, Schlinkert R, Sarr MG. The natural history of surgically treated primary adenocarcinoma of the appendix. Ann Surg. 1994;219(1):51-7.

14. Cortina R, McCormick J, Kolm P, Perry RR. Management and prognosis of adenocarcinoma of the appendix. Dis Colon Rectum. 1995;38(8):848-52.

15. Lyss AP. Appendiceal malignancies. Semin Oncol. 1988;15(2):129-37.

16. Overman MJ, Fournier K, Hu CY, Eng C, Taggart M, Royal R, Mansfield P, Chang GJ. Improving the AJCC/TNM staging for adenocarcinomas of the appendix: the prognostic impact of histological grade. Ann Surg. 2013; 257(6):1072-8.

17. Blum Murphy MA, Elimova E, Ajani JA. Current concepts and future potential in neoadjuvant chemotherapy for esophageal cancer. Expert Rev Gastroenterol Hepatol. 2016;10(3):383-92.

18. Harada K, Mizrak Kaya D, Shimodaira Y, Ajani JA. Global chemotherapy development for gastric cancer. Gastric Cancer. 2017; 20(Suppl 1):92-101.

19. Turaga K, Levine E, Barone R, Sticca R, Petrelli N, Lambert L, Nash G, Morse M, Adbel-Misih R, Alexander HR, et al. Consensus guidelines from the American Society of Peritoneal Surface Malignancies on standardizing the delivery of hyperthermic intraperitoneal chemotherapy (HIPEC) in colorectal cancer patients in the United States. Ann Surg Oncol. 2014;21(5):1501-5.

20. Asare EA, Compton CC, Hanna NN, Kosinski LA, Washington MK, Kakar S, Weiser MR, Overman MJ. The impact of stage, grade, and mucinous histology on the efficacy of systemic chemotherapy in adenocarcinomas of the appendix: analysis of the national cancer data base. Cancer. 2016;122(2): 213-21.

21. Xie X, Zhou Z, Song Y, Li W, Diao D, Dang C, Zhang H. The management and prognostic prediction of adenocarcinoma of appendix. Sci Rep. 2016;6: 39027.

\section{Publisher's Note}

Springer Nature remains neutral with regard to jurisdictional claims in published maps and institutional affiliations.
Ready to submit your research? Choose BMC and benefit from:

- fast, convenient online submission

- thorough peer review by experienced researchers in your field

- rapid publication on acceptance

- support for research data, including large and complex data types

- gold Open Access which fosters wider collaboration and increased citations

- maximum visibility for your research: over $100 \mathrm{M}$ website views per year

At $\mathrm{BMC}$, research is always in progress.

Learn more biomedcentral.com/submissions 